\title{
On Perturbation Solutions for Axisymmetric Bending Boundary Values of a Deep Thin Spherical Shell
}

\author{
Rong Xiao, ${ }^{1,2}$ Danhui Dan, ${ }^{1}$ and Wei Cheng ${ }^{1}$ \\ ${ }^{1}$ Department of Bridge Engineering, Tongji University, Room 711, Bridge Building, 1239 Siping Road, Shanghai 200092, China \\ ${ }^{2}$ Shanghai Municipal Engineering Design Institute (Group) Co., Ltd., 901 North Zhongshan Road (2nd), Shanghai 200092, China \\ Correspondence should be addressed to Danhui Dan; dandanhui@tongji.edu.cn
}

Received 4 July 2014; Accepted 8 August 2014; Published 31 August 2014

Academic Editor: Peter Liu

Copyright (c) 2014 Rong Xiao et al. This is an open access article distributed under the Creative Commons Attribution License, which permits unrestricted use, distribution, and reproduction in any medium, provided the original work is properly cited.

\begin{abstract}
On the basis of the general theory of elastic thin shells and the Kirchhoff-Love hypothesis, a fundamental equation for a thin shell under the moment theory is established. In this study, the author derives Reissner's equation with a transverse shear force $Q_{1}$ and the displacement component $w$. These basic unknown quantities are derived considering the axisymmetry of the deep, thin spherical shell and manage to constitute a boundary value question of axisymmetric bending of the deep thin spherical shell under boundary conditions. The asymptotic solution is obtained by the composite expansion method. At the end of this paper, to prove the correctness and accuracy of the derivation, an example is given to compare the numerical solution by ANSYS and the perturbation solution. Meanwhile, the effects of material and geometric parameters on the nonlinear response of axisymmetric deep thin spherical shell under uniform external pressure are also analyzed in this paper.
\end{abstract}

\section{Introduction}

Thin shells are widely used in engineering structures, especially in long-span structures, for their good performance in strength and stiffness. Thin shells are also used in some mechanisms as the stressed surface which is applied distributed pressure like water and wind pressure. Recently, a novelty wind pressure sensor is proposed by Dan et al. [1], which used fiber Bragg grating as the transducer and the thin shell as the wind pressure bearing component. The motivation of this paper is to find an analytical approach to analyse the internal force and deformation behaviors of the wind pressure stressed thin shell in the sensor.

At present, the research on the mechanical behavior of deep thin spherical shells is as common as that of thin circular plates or shallow spherical shells. In 1948, Weichang [2] first applied the thin film expansion method to obtain the perturbation solution to large deflections of clamped thin isotropic circular plates under uniformly distributed transverse loads. In 1967, Lukasiewicz, [3] with the shallow shell theory, solved the situation when a shallow shell bore a concentrated tangential force at its vertex, a concentrated couple in the normal plane, or a concentrated couple in the tangential plane. Huy Bich and Van Tung (see [4]) proposed an analytical approach to investigate the nonlinear axisymmetric response of functionally graded shallow spherical shells subjected to uniform external pressure incorporating the effects of temperature. Several authors $[5,6]$ used some assumptions to obtain the analytical solutions of the problem for both shallow and deep but isotropic spherical shells. Li et al. [7] adopted the modified iteration method to solve nonlinear stability problem of shear deformable isotropic shallow spherical shells under uniform external pressure.

Deep thin spherical shell bending is generally complicated. However, as is commonly seen in real engineering applications, the problems for axisymmetric bending are easily solved. In a literature example [8], the equation can be solved with Reissner's equations $[9,10]$ describing axially symmetric deformations of thin elastic deep shells of revolution by large deflections and rotation angles by adding some boundary conditions, but without giving the specific realization approach. Evkin and Kalamkarov [11, 12] 
applied the singular perturbation method in combination with the variational method to the general Reissner's equations. Thredgold [13] considered the numerical solution of equations for the deformation of a spherical shell using a finite difference methodology. Colgan et al. [14] used an elementary perturbation analysis to find an analytic solution to the linear equations for the small symmetric deformation of a deep spherical shell. Evkin [15] studied nonlinear behavior of deep orthotropic spherical shells under inward radial concentrated load, and the asymptotic solution he obtained is in close agreement with the experimental and numerical results and has the same accuracy (in the asymptotic meaning) as the given equations of nonlinear theory of thin shells. Some domestic researchers [16-18] solved the bending problem of deep thin spherical shells by establishing a differential equation with the assumption that the mid-surface meridian displacement $u=0$ under the edge effect. The accuracy of solutions is proven afterwards. Some researchers apply the nonmoment theory, considering the edge effect in solution, with reference to the solution of cylinder shell. This method should be based on the assumption that both bending moment and torque are zero on the transversal surface. In addition, three more conditions should be satisfied compulsively to increase applicability. First, the middle surface of the shell must be a curved smooth surface without any abrupt changes of slope or curvature. Second, the external load must be continuously distributed with neither an abrupt change nor a concentrated load. Third, the deflection on the edge cannot be constrained, nor can the rotation around the boundary line be constrained. In real applications, the first two requirements are easily satisfied, but the third is generally difficult. In some areas near the shell's boundary, moment is almost inevitable. All of these cause an adverse effect on accuracy and feasibility. Therefore, it is necessary to find a new analysis method that is applicable to the real stress state. We also noticed that the advanced composite materials and structure are hot issues and are studied by many researchers nowadays. Recently, new advanced shear deformation theories are developed by El Meiche et al. [19] to analyse the force and deformation behaviors of thin sandwich plate, and its efficient and higher-order version is also studied by Larbi et al. [20] and Bessaim et al. [21]. This shear deformation theories based approach is proved to be a good performance on the static and dynamical analysis of plate-like structure. It may be a worthy task to apply this approach to thin shell-like structures. Here in this paper, we employ the moment theory to determine the deformation behavior of a thin shell under pressure.

Based on the general theory of an elastic thin shell, a fundamental equation for thin shells under the moment theory is directly established. Reissner's equation is derived with a transverse shear force $Q_{1}$ and displacement component $w$ as the basic unknown quantities, considering the axisymmetry of the deep thin spherical shell. We also manage to constitute a boundary value question of axisymmetric bending of deep thin spherical shells under boundary conditions. With perturbation theory based on the composite expansion method, a solution with a higher accuracy is obtained.

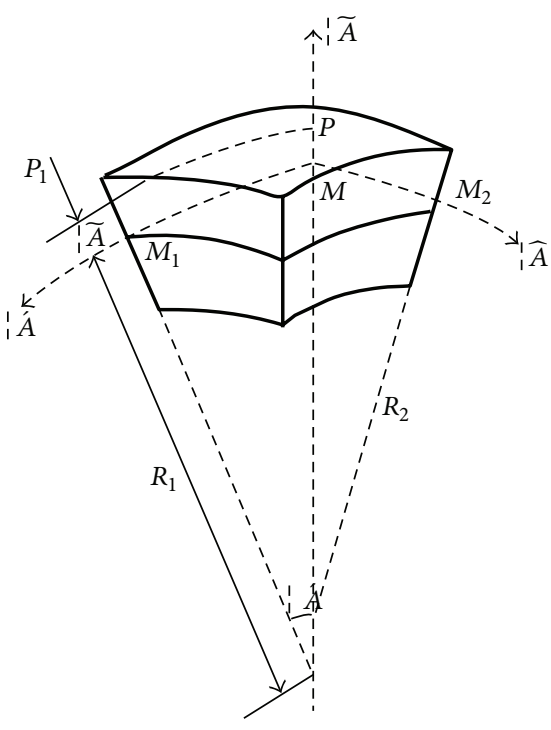

FIgURE 1: Orthogonal curvilinear coordinate system on the middle surface of a deep thin spherical shell.

\section{Fundamental Equation of Deep Thin Spherical Shell}

As shown in Figure 1, the radius is $R$, the central angle is $\alpha_{0}$, the thickness is $t$, and the principal radius of curvature in the middle surface is $R_{1}=R_{2}=R$. The Lame coefficients in the middle surface, $H_{1}, H_{2}$ in Figure 1 , are $H_{1}=R$ and $H_{2}=R \sin \alpha$, respectively. An orthogonal curvilinear coordinate system $\alpha \beta \gamma$ is built. As shown in Figure 1, $\alpha$ and $\beta$ are the principal curves of curvature. $\gamma$ is a straight normal line pointing towards the convex side.

2.1. Geometric Equation. The basic hypothesis of shell theory is adopted. The normal in the middle is perpendicular to the middle surface even after it is deformed. The line, which is perpendicular to the middle surface, is trivial.

The normal strains $\varepsilon_{1}, \varepsilon_{2}$ of the points in the middle surface along the $\alpha$ and $\beta$ directions are as follows:

$$
\begin{aligned}
& \varepsilon_{1}=\frac{1}{R}\left(\frac{d u}{d \alpha}+w\right), \\
& \varepsilon_{2}=\frac{1}{R}(u \cot \alpha+w),
\end{aligned}
$$

where $u$ and $w$ are the displacements of the points on the middle surface going through $\alpha$ and $\gamma$, respectively.

The changes of their principal curvatures $\chi_{1}$ and $\chi_{2}$ are as follows:

$$
\begin{aligned}
& \chi_{1}=-\frac{1}{R^{2}} \frac{d^{2} w}{d \alpha^{2}} \\
& \chi_{2}=-\frac{1}{R^{2}} \frac{d w}{d \alpha} \cot \alpha
\end{aligned}
$$


The strains $e_{1}$ and $e_{2}$ of any points in the shell can be expressed as follows:

$$
\begin{aligned}
& e_{1}=\varepsilon_{1}+\chi_{1} \gamma, \\
& e_{2}=\varepsilon_{2}+\chi_{2} \gamma .
\end{aligned}
$$

For a deep thin spherical shell, the components of the strain that reflect the whole shell's deformation status are obtained according to (3).

2.2. Physics Equation. On the basis of the basic hypothesis of shell theory, the normal stress parallel to the middle surface's cross section is far less than the normal stress on the cross section. Regardless of its effect on the strain, the following physics equation is obtained:

$$
\begin{aligned}
& \sigma_{1}=\frac{E}{1-\mu^{2}}\left(e_{1}+\mu e_{2}\right), \\
& \sigma_{2}=\frac{E}{1-\mu^{2}}\left(e_{2}+\mu e_{1}\right) .
\end{aligned}
$$

The internal forces $N_{1}, N_{2}, M_{1}$, and $M_{2}$ can be described by the mid-surface deformation component as below:

$$
\begin{aligned}
& N_{1}=\frac{E t}{1-\mu^{2}}\left(\varepsilon_{1}+\mu \varepsilon_{2}\right), \\
& N_{2}=\frac{E t}{1-\mu^{2}}\left(\varepsilon_{2}+\mu \varepsilon_{1}\right), \\
& M_{1}=D\left(\chi_{1}+\mu \chi_{2}\right), \\
& M_{2}=D\left(\chi_{2}+\mu \chi_{1}\right) .
\end{aligned}
$$

In (4) and (5), $\mu$ is Poisson's ratio of the shell; $D$ is flexural rigidity, $D=E t^{3} / 12\left(1-\mu^{2}\right)$.

According to (5), $\varepsilon_{1}+v \varepsilon_{2}, \varepsilon_{2}+v \varepsilon_{1}, \chi_{1}+v \chi_{2}$, and $\chi_{2}+v \chi_{1}$, then the following formula is obtained:

$$
\begin{aligned}
& \sigma_{1}=\frac{N_{1}}{h}+\frac{12 M_{1}}{h^{3}} \gamma, \\
& \sigma_{2}=\frac{N_{2}}{h}+\frac{12 M_{2}}{h^{3}} \gamma .
\end{aligned}
$$

From the equations above, it can be seen that, in the shell, the stresses caused by film forces $N_{1}$ and $N_{2}$ are uniformly distributed along the shell thickness. The bending stresses caused by bending moments $M_{1}$ and $M_{2}$ are distributed in a straight line along the shell thickness.

2.3. Equilibrium Equation. By converting the external load into load components $X$ and $Z$ per unit area of middle surface, the following differential equilibrium is obtained by listing all the internal force components projecting along $\alpha$ and $\gamma$ and the projection of axial moment along $\alpha$ and $\gamma$ :

$$
\begin{aligned}
& \frac{d N_{1}}{d \alpha}+\left(N_{1}-N_{2}\right) \cot \alpha+Q_{1}+X R=0, \\
& \frac{d Q_{1}}{d \alpha}+Q_{1} \cot \alpha-\left(N_{1}+N_{2}\right)+Z R=0, \\
& \frac{d M_{1}}{d \alpha}+\left(M_{1}-M_{2}\right) \cot \alpha-Q_{1} R=0 .
\end{aligned}
$$

\section{Perturbation Solution for Axisymmetric Bending Boundary Value}

By substituting geometric equations (1) and (2) into the physics equation (5), the elastic equation describing the relationship between internal force and displacement is obtained:

$$
\begin{aligned}
& \frac{d u}{d \alpha}+w=\frac{R}{E t}\left(N_{1}-\mu N_{2}\right), \\
& u \cot \alpha+w=\frac{R}{E t}\left(N_{2}-\mu N_{1}\right), \\
& M_{1}=-\frac{D}{R^{2}}\left(\frac{d^{2} w}{d \alpha^{2}}+\mu \frac{d w}{d \alpha} \cot \alpha\right), \\
& M_{2}=-\frac{D}{R^{2}}\left(\frac{d w}{d \alpha} \cot \alpha+\mu \frac{d^{2} w}{d \alpha^{2}}\right) .
\end{aligned}
$$

Elastic equation (8) and the three equilibrium equations (7) together with the boundary conditions constitute the boundary value problem of axisymmetric bending of a deep thin spherical shell. Seven unknown functions can be solved in total, including 5 internal forces $N_{1}, N_{2}, M_{1}, M_{2}$, and $Q_{1}$ and 2 displacements $u$ and $w$.

3.1. Formula for Mid-Surface Meridian Displacement $u$ under the Moment Theory. By subtracting the first two formulas of (8), $w$ is eliminated, and the following formula is obtained:

$$
\frac{d u}{d \alpha}-u \cot \alpha=\frac{(1+\mu) R}{E h}\left(N_{1}-N_{2}\right) .
$$

By substituting variable $u=\zeta \sin \alpha$, (9) is changed into the following form:

$$
\frac{d \zeta}{d \alpha}=\frac{(1+\mu) R}{E h \sin \alpha}\left(N_{1}-N_{2}\right) .
$$

After integration, the original variable is substituted into the equation

$$
u=\frac{(1+\mu) R}{E h} \sin \alpha \int_{0}^{\alpha} \frac{\left(N_{1}-N_{2}\right)}{\sin \alpha} d \alpha+C .
$$

According to the symmetry condition of a shell roof, $\left.u\right|_{\alpha=0}=0, d u /\left.d \alpha\right|_{\alpha=0}=0$.

When $\alpha=0, C=0$, the formula below is obtained:

$$
u=\frac{(1+\mu) R}{E h} \sin \alpha \int_{0}^{\alpha} \frac{\left(N_{1}-N_{2}\right)}{\sin \alpha} d \alpha .
$$


3.2. Reissner's Equation. Based on the equilibrium equation (7), the internal forces $N_{1}, N_{2}$ can be described as follows:

$$
\begin{aligned}
N_{1} & =Q_{1} \cot \alpha+\frac{R}{\sin ^{2} \alpha} \int_{0}^{\alpha}(Z \cos \alpha-X \sin \alpha) \sin \alpha d \alpha \\
& =Q_{1} \cot \alpha+N_{1}^{*}, \\
N_{2} & =\frac{d Q_{1}}{d \alpha}+Z R-\frac{R}{\sin ^{2} \alpha} \int_{0}^{\alpha}(Z \cos \alpha-X \sin \alpha) \sin \alpha d \alpha \\
& =\frac{d Q_{1}}{d \alpha}+N_{2}^{*},
\end{aligned}
$$

where

$$
\begin{aligned}
& N_{1}^{*}=\int_{0}^{\alpha}(Z \cos \alpha-X \sin \alpha) \sin \alpha d \alpha, \\
& N_{2}^{*}=Z R-\int_{0}^{\alpha}(Z \cos \alpha-X \sin \alpha) \sin \alpha d \alpha .
\end{aligned}
$$

The subscript $*$ represents the displacement and internal forces under the nonmoment theory.

Now a differential equation can be derived with Reissner's approach.

The last two equations are substituted into the third equation of equilibrium equation (7):

$$
\frac{d^{3} w}{d \alpha^{3}}+\frac{d^{2} w}{d \alpha^{2}} \cot \alpha-\frac{d w}{d \alpha}\left(\cot ^{2} a+\mu\right)=-\frac{R^{3}}{D} Q_{1}
$$

When edge effect works, $u_{l}=0^{[7]}$, and the following equation can be obtained by subtracting the second equation from the first in elastic equation (8):

$$
(1+\mu)\left(N_{1}-N_{2}\right)+\frac{E t}{R} u^{*} \cot \alpha-\frac{E t}{R} \frac{d u^{*}}{d \alpha}=0
$$

By taking derivative of (8),

$$
\frac{E t}{R} \frac{d w}{d \alpha}=\frac{d}{d \alpha}\left(N_{2}-\mu N_{1}\right)-\frac{E t}{R} \frac{d}{d \alpha}\left(u^{*} \cot \alpha\right) .
$$

According to (18) and (17), the following formula is obtained:

$\frac{E t}{R} \frac{d w}{d \alpha}=\frac{d}{d \alpha}\left(N_{2}-\mu N_{1}\right)-(1+\mu)\left(N_{1}-N_{2}\right) \cot \alpha+\frac{E t}{R} u^{*}$.

By substituting (12) and (13) into (19), we have

$$
\begin{aligned}
\frac{d^{2} Q_{1}}{d \alpha^{2}}+ & \frac{d Q_{1}}{d \alpha} \cot \alpha-Q_{1}\left(\cot ^{2} \alpha-\mu\right) \\
= & \frac{E t}{R} \frac{d w}{d \alpha}-\frac{d}{d \alpha}\left(N_{2}^{*}-\mu N_{1}^{*}\right) \\
& -(1+\mu) \frac{d}{d \alpha}\left[\cos \alpha \int_{0}^{\alpha} \frac{\left(N_{2}^{*}-N_{1}^{*}\right)}{\sin \alpha} d \alpha\right] .
\end{aligned}
$$

Then the differential equation of deep thin spherical shell is obtained:

$$
\begin{aligned}
\frac{d^{3} w}{d \alpha^{3}}+ & \frac{d^{2} w}{d \alpha^{2}} \cot \alpha-\frac{d w}{d \alpha}\left(\cot ^{2} \alpha+\mu\right)=-\frac{R^{3}}{D} Q_{1}, \\
\frac{d^{2} Q_{1}}{d \alpha^{2}}+ & \frac{d Q_{1}}{d \alpha} \cot \alpha-Q_{1}\left(\cot ^{2} \alpha-\mu\right) \\
= & \frac{E t}{R} \frac{d w}{d \alpha}-\frac{d}{d \alpha}\left(N_{2}^{*}-\mu N_{1}^{*}\right) \\
& -(1+\mu) \frac{d}{d \alpha}\left[\cos \alpha \int_{0}^{\alpha} \frac{\left(N_{2}^{*}-N_{1}^{*}\right)}{\sin \alpha} d \alpha\right] .
\end{aligned}
$$

We chose $w^{*}$ as the particular solution of (21), and its homogeneous equation can be derived as a perturbation solution:

$$
\begin{aligned}
& \frac{d^{3} w}{d \alpha^{3}}+\frac{d^{2} w}{d \alpha^{2}} \cot \alpha-\frac{d w}{d \alpha}\left(\cot ^{2} \alpha+\mu\right)=-\frac{R^{3}}{D} Q_{1} \\
& \frac{d^{2} Q_{1}}{d \alpha^{2}}+\frac{d Q_{1}}{d \alpha} \cot \alpha-Q_{1}\left(\cot ^{2} \alpha-\mu\right)=\frac{E t}{R} \frac{d w}{d \alpha}
\end{aligned}
$$

3.3. Perturbation Solution to Boundary Problem. The fixed support deep thin spherical shell is subjected to a uniformly distributed normal load. This can be used as an example to obtain its internal force by the perturbation method.

In regard to an axisymmetric deformation of a bottom fixed deep thin spherical shell, the boundary conditions are as follows:

$$
\begin{gathered}
\left.w\right|_{\alpha=\alpha_{0}}=0,\left.\quad \frac{d w}{d \alpha}\right|_{\alpha=\alpha_{0}}=0, \quad \lim _{\alpha \rightarrow 0} w=\lim _{\alpha \rightarrow 0} w^{*}=\alpha^{\prime}, \\
\lim _{\alpha \rightarrow 0} \frac{d w}{d \alpha}=\lim _{\alpha \rightarrow 0} \frac{d w^{*}}{d \alpha}=0, \quad \lim _{\alpha \rightarrow 0} Q_{1}=0 .
\end{gathered}
$$

After variable substitution, $\widetilde{Q_{1}}=Q_{1} \sin \alpha, \varphi=\alpha_{0}-\alpha$, $d \varphi=-d \alpha, x=\cos \left(\alpha_{0}-\varphi\right) / \cos \alpha_{0}$, and $a=1 / \cos \alpha_{0}, a>1$, (22) can be changed into

$$
\begin{gathered}
\left(x^{2}-a^{2}\right)^{2} \frac{d^{3} w}{d x^{3}}+4 x\left(x^{2}-a^{2}\right) \frac{d^{2} w}{d x^{2}} \\
+(1+\mu)\left(x^{2}-a^{2}\right) \frac{d w}{d x}=\frac{R^{3}}{D} a \widetilde{Q_{1}}, \\
a\left(x^{2}-a^{2}\right) \frac{d^{2} \widetilde{Q_{1}}}{d x^{2}}-a(1+\mu) \widetilde{Q_{1}}=-\frac{E t}{R}\left(x^{2}-a^{2}\right) \frac{d w}{d x} .
\end{gathered}
$$

Boundary conditions are changed into

$$
\begin{gathered}
\left.w\right|_{x=1}=0,\left.\quad \frac{d w}{d x}\right|_{x=1}=0, \quad \lim _{x \rightarrow a} w=\lim _{x \rightarrow a} w^{*}=\alpha^{\prime}, \\
\lim _{x \rightarrow a} \frac{d w}{d x}=\lim _{x \rightarrow a} \frac{d w^{*}}{d x}=0, \quad \lim _{x \rightarrow a} \widetilde{Q_{1}}=0 .
\end{gathered}
$$


Consider $R \gg t$ and let $\varepsilon=\sqrt{t / \sqrt{12\left(1-\mu^{2}\right)}} R$, and nondimensionalization is carried out:

$$
Q=\sqrt{\frac{\sqrt{12\left(1-\mu^{2}\right)} R}{t}} \frac{R \widetilde{Q_{1}}}{E t^{2}}, \quad W=\frac{w}{t} .
$$

Finally, the differential equation (24) can be written in the following form:

$$
\begin{gathered}
\varepsilon^{3}\left(x^{2}-a^{2}\right)^{2} \frac{d^{3} W}{d x^{3}}+\varepsilon^{3} 4 x\left(x^{2}-a^{2}\right) \frac{d^{2} W}{d x^{2}} \\
+\varepsilon^{3}(1+\mu)\left(x^{2}-a^{2}\right) \frac{d W}{d x}=a Q, \\
\varepsilon a\left(x^{2}-a^{2}\right) \frac{d^{2} Q}{d x^{2}}-\varepsilon a(1+\mu) Q=-\left(x^{2}-a^{2}\right) \frac{d W}{d x} .
\end{gathered}
$$

The boundary conditions are changed into

$$
\begin{gathered}
\left.W\right|_{x=1}=0,\left.\quad \frac{d W}{d x}\right|_{x=1}=0, \quad \lim _{x \rightarrow a} W=\frac{\alpha^{\prime}}{t}=\alpha^{\prime \prime}, \\
\lim _{x \rightarrow a} \frac{d W}{d x}=\lim _{x \rightarrow a} \frac{d W^{*}}{d x}=0, \quad \lim _{x \rightarrow a} Q=0 .
\end{gathered}
$$

By employing the composite expansion method, the perturbation solution to the differential equation is set as follows:

$$
\begin{gathered}
W(x, \varepsilon)=F(x, \varepsilon)+K(y, \varepsilon), \\
Q(x, \varepsilon)=J(x, \varepsilon)+\Omega(y, \varepsilon) .
\end{gathered}
$$

Generally, a differential equation has two types of solutions: an exterior solution and an interior solution. The counterparts are then matched. In the composite expansion method, the perturbation solutions $W(x, \varepsilon)$ and $Q(x, \varepsilon)$ are divided into two parts, $F(x, \varepsilon)$ and $K(y, \varepsilon)$. Then the differential equations that $F(x, \varepsilon), K(y, \varepsilon), J(x, \varepsilon)$, and $\Omega(y, \varepsilon)$ should satisfy are deduced, respectively. The benefit of this approach is that the boundary conditions can be strictly met.

The boundary conditions of the perturbation solution to the equation $y=(x-1) / \varepsilon$ can be rewritten as follows:

$$
\begin{gathered}
\lim _{x \rightarrow a} Q(x, \varepsilon)=\lim _{x \rightarrow a} J(x, \varepsilon)+\lim _{y \rightarrow \infty} \Omega(y, \varepsilon)=0, \\
\lim _{x \rightarrow a} W(x, \varepsilon)=\lim _{x \rightarrow a} F(x, \varepsilon)+\lim _{y \rightarrow \infty} K(y, \varepsilon)=\alpha^{\prime \prime}, \\
\left.W(x, \varepsilon)\right|_{x=1}=\left.F(x, \varepsilon)\right|_{x=1}+\left.K(y, \varepsilon)\right|_{y=0}=0, \\
\left.Q(x, \varepsilon)\right|_{x=1}=\left.J(x, \varepsilon)\right|_{x=1}+\left.\Omega(y, \varepsilon)\right|_{y=0}=0 .
\end{gathered}
$$

It is required that $K_{0}(y, \varepsilon), K_{1}(y, \varepsilon), \ldots, \Omega_{0}(y, \varepsilon), \Omega_{1}(y, \varepsilon)$, $\ldots$ are shape functions of boundary layer, of which the values beyond the boundary can be ignored. Hence, when $\eta \rightarrow \infty$, the function approaches zero exponentially; that is,

$$
\lim _{y \rightarrow \infty} K(y, \varepsilon)=0, \quad \lim _{y \rightarrow \infty} \Omega(y, \varepsilon)=0 .
$$

Thus,

$$
\lim _{x \rightarrow a} F(x, \varepsilon)=\alpha^{\prime \prime}, \quad \lim _{x \rightarrow a} J(x, \varepsilon)=0 .
$$

By substituting $F(x, \varepsilon)$ and $J(x, \varepsilon)$ into (27), the following equation set is obtained:

$$
\begin{gathered}
\varepsilon^{3}\left(x^{2}-a^{2}\right)^{2} \frac{d^{3} F}{d x^{3}}+\varepsilon^{3} 4 x\left(x^{2}-a^{2}\right) \frac{d^{2} F}{d x^{2}} \\
+\varepsilon^{3}(1+\mu)\left(x^{2}-a^{2}\right) \frac{d F}{d x}=x J, \\
\varepsilon a\left(x^{2}-a^{2}\right) \frac{d^{2} J}{d x^{2}}-\varepsilon a(1+\mu) J=-\left(x^{2}-a^{2}\right) \frac{d F}{d x} .
\end{gathered}
$$

Let the solution in (33) take the forms of

$$
\begin{gathered}
F=F_{0}+\varepsilon F_{1}+\varepsilon^{2} F_{2}+\cdots, \\
J=J_{0}+\varepsilon J_{1}+\varepsilon^{2} J_{2}+\cdots
\end{gathered}
$$

And substituting it into (33) and comparing the coefficients of $\varepsilon^{n}$ under $n=0,1,2, \ldots$, we obtain

$$
J(x, \varepsilon)=0, \quad \frac{d F(x, \varepsilon)}{d x}=0 .
$$

Thus,

$$
\left.K(y, \varepsilon)\right|_{y=0}=-\alpha^{\prime \prime},\left.\quad \Omega(y, \varepsilon)\right|_{y=0}=0 .
$$

By substituting $x=\varepsilon y+1, K(y, \varepsilon)$, and $\Omega(y, \varepsilon)$ into (33), the following formula is obtained:

$$
\begin{gathered}
(1+\mu)\left[\varepsilon^{4} y^{2}+2 \varepsilon^{3} y+\left(1-a^{2}\right) \varepsilon^{2}\right] \frac{d K}{d y} \\
+\left[4 \varepsilon^{4} y^{3}+12 \varepsilon^{3} y^{2}+\left(12-4 a^{2}\right) \varepsilon^{2} y+4\left(1-a^{2}\right) \varepsilon\right] \frac{d^{2} K}{d y^{2}} \\
+\left[\varepsilon^{4} y^{4}+4 \varepsilon^{3} y^{3}+\left(6-2 a^{2}\right) \varepsilon^{2} y^{2}+4\left(1-a^{2}\right) \varepsilon y\right. \\
\left.+\left(1-a^{2}\right)^{2}\right] \frac{d^{3} K}{d y^{3}}=a \Omega, \\
a\left[\varepsilon^{2} y^{2}+2 \varepsilon y+\left(1-a^{2}\right)\right] \frac{d^{2} \Omega}{d y^{2}}-\varepsilon^{2} a(1+\mu) \Omega \\
=-\left[\varepsilon^{2} y^{2}+2 \varepsilon y+\left(1-a^{2}\right)\right] \frac{d K}{d y} .
\end{gathered}
$$

The boundary conditions of the corresponding situations are $\left.K\right|_{y=0}=-\alpha^{\prime \prime}, d K /\left.d y\right|_{y=0}=0, \lim _{y \rightarrow \infty} K=0$, $\lim _{y \rightarrow \infty}(d K / d y)=0$, and $\lim _{y \rightarrow \infty} \Omega=0$. 
To ensure the accuracy, the perturbation solutions are approximated as follows.

(1) $n=0$. To substitute $K=K_{0}+0(\varepsilon)$ and $\Omega=\Omega_{0}+0(\varepsilon)$ into (37) and compare their coefficients of terms $\varepsilon^{0}$, we have

$$
\begin{gathered}
\left(1-a^{2}\right)^{2} \frac{d^{3} K_{0}}{d y^{3}}=a \Omega_{0}, \\
a\left(1-a^{2}\right) \frac{d^{2} \Omega_{0}}{d y^{2}}=-\left(1-a^{2}\right) \frac{d K_{0}}{d y} .
\end{gathered}
$$

Let $m=\left[1 / 4\left(1-a^{2}\right)^{2}\right]^{1 / 4} ;(38)$ can be changed into

$$
\frac{d^{5} K_{0}}{d y^{5}}+4 m^{4} \frac{d K_{0}}{d y}=0
$$

The general solution of (39) is

$$
\begin{aligned}
K_{0}= & e^{-m y}\left(A_{1} \cos m y+A_{2} \sin m y\right) \\
& +e^{b \eta}\left(A_{3} \cos m y+A_{4} \sin m y\right)+A_{5} .
\end{aligned}
$$

By applying the boundary conditions,

$$
\begin{gathered}
\left.K_{0}\right|_{y=0}=-\alpha^{\prime \prime},\left.\quad \frac{d K_{0}}{d y}\right|_{y=0}=0, \quad \lim _{y \rightarrow \infty} K_{0}=0, \\
\lim _{y \rightarrow \infty} \frac{d K_{0}}{d y}=0, \quad \lim _{y \rightarrow \infty} \Omega_{0}=0,
\end{gathered}
$$

we get the following constant:

$$
A_{1}=A_{2}=-\alpha^{\prime \prime}, \quad A_{3}=A_{4}=A_{5}=0 .
$$

The particular solution to (38) that meets the same boundary conditions above can be written as

$$
\begin{gathered}
K_{0}=-\alpha^{\prime \prime} e^{-m y}(\cos m y+\sin m y), \\
\Omega_{0}=-b \alpha^{\prime \prime} e^{-m y} \cos m y,
\end{gathered}
$$

where $b=\sqrt{2 /\left(2 m^{2}+1\right)}$.

(2) $n=1$. To substitute $K=K_{0}+\varepsilon K_{1}+0(\varepsilon)$ and $\Omega=\Omega_{0}+$ $\varepsilon \Omega_{1}+0(\varepsilon)$ into (37) and compare their coefficients of terms $\varepsilon$, we get

$$
\begin{aligned}
& \left(1-a^{2}\right)^{2} \frac{d^{3} K_{1}}{d y^{3}} \\
& =a \Omega_{1}-4\left(1-a^{2}\right) y \frac{d^{3} K_{0}}{d y^{3}}-4\left(1-a^{2}\right) \frac{d^{2} K_{0}}{d y^{2}}, \\
& a\left(1-a^{2}\right) \frac{d^{2} \Omega_{1}}{d y^{2}} \\
& =-\left(1-a^{2}\right) \frac{d K_{1}}{d y}-2 a y \frac{d^{2} \Omega_{0}}{d y^{2}}-2 y \frac{d K_{0}}{d y} .
\end{aligned}
$$

The particular solution to (44) which meets the boundary condition is

$$
\begin{aligned}
& K_{1}=2 m^{3} \alpha^{\prime \prime} y^{2} e^{-m y} \sin m y-m^{2} \alpha^{\prime \prime} y e^{-m y}(\cos m y+\sin m y), \\
& \Omega_{1}= b m^{3} \alpha^{\prime \prime} y^{2} e^{-m y}(\cos m y+\sin m y) \\
&+b m^{2} \alpha^{\prime \prime} y e^{-m y} \cos m y \\
&+\frac{b m}{2} \alpha^{\prime \prime} e^{-m y}(\cos m y-\sin m y) .
\end{aligned}
$$

(3) $n=2$. To substitute $K=K_{0}+\varepsilon K_{1}+\varepsilon^{2} K_{2}+0(\varepsilon)$ and $\Omega=$ $\Omega_{0}+\varepsilon \Omega_{1}+\varepsilon^{2} \Omega_{2}+0(\varepsilon)$ into (37) and compare the coefficients of terms $\varepsilon^{2}$, we have

$$
\begin{aligned}
(1- & \left.a^{2}\right)^{2} \frac{d^{3} K_{2}}{d y^{3}} \\
= & a \Omega_{2}-4\left(1-a^{2}\right) y \frac{d^{3} K_{1}}{d y^{3}}-\left(6-2 a^{2}\right) y^{2} \frac{d^{3} K_{0}}{d y^{3}} \\
& -4\left(1-a^{2}\right) \frac{d^{2} K_{1}}{d y^{2}}-\left(12-4 a^{2}\right) y \frac{d^{2} K_{0}}{d y^{2}}-\left(1-a^{2}\right) \frac{d K_{0}}{d y},
\end{aligned}
$$$$
a\left(1-a^{2}\right) \frac{d^{2} \Omega_{2}}{d y^{2}}
$$$$
\begin{aligned}
= & -\left(1-a^{2}\right) \frac{d K_{2}}{d y}-2 a y \frac{d^{2} \Omega_{1}}{d y^{2}}-a y^{2} \frac{d^{2} \Omega_{0}}{d y^{2}} \\
& +a(1+\mu) \Omega_{0}-2 y \frac{d K_{1}}{d y}-y^{2} \frac{d K_{0}}{d y} .
\end{aligned}
$$

Its particular solution can be written as

$$
\begin{aligned}
K_{2}= & m^{6} \alpha^{\prime \prime} y^{4} e^{-m y}(\cos m y-\sin m y) \\
& +\left(6 m^{5}+\frac{2}{3} m^{3}\right) \alpha^{\prime \prime} y^{3} e^{-m y} \sin m y \\
& -\left(\frac{5}{2} m^{4}+\frac{1}{2} m^{2}\right) \alpha^{\prime \prime} y^{2} e^{-m y}(\cos m y+\sin m y) \\
& -\left[\frac{1}{2} m^{3}+m+\frac{1}{2}(1+\mu) m\right] \alpha^{\prime \prime} y e^{-m y} \cos m y, \\
\Omega_{2}= & -b m^{6} \alpha^{\prime \prime} e^{-m y} y^{4} \sin m y \\
& +b\left(m^{5}+\frac{1}{3} m^{3}\right) \alpha^{\prime \prime} y^{3} e^{-m y}(\cos m y+\sin m y) \\
& +\frac{b}{2}\left(m^{4}+m^{2}\right) \alpha^{\prime \prime} y^{2} e^{-m y} \cos m y \\
& +\frac{b}{4}\left[m^{3}-(1+\mu) m\right] \alpha^{\prime \prime} y e^{-m y}(\cos m y-\sin m y) \\
& -\frac{b}{4}\left[m^{2}+3(1+\mu)\right] \alpha^{\prime \prime} e^{-m y} \sin m y .
\end{aligned}
$$


Thus, the second order perturbation solution to differential equation (27) is obtained as shown below:

$$
\begin{gathered}
W=F+K_{0}+\varepsilon K_{1}+\varepsilon^{2} K_{2}+O\left(\varepsilon^{3}\right), \\
Q=\Omega_{0}+\varepsilon \Omega_{1}+\varepsilon^{2} \Omega_{2}+O\left(\varepsilon^{3}\right) .
\end{gathered}
$$

The solution to the differential equation is

$$
\begin{gathered}
w \sim t\left(F+K_{0}+\varepsilon K_{1}+\varepsilon^{2} K_{2}\right), \\
Q_{1} \sim \frac{\varepsilon E t^{2}}{R \sin \alpha}\left(\Omega_{0}+\varepsilon \Omega_{1}+\varepsilon^{2} \Omega_{2}\right) .
\end{gathered}
$$

The following perturbation solutions of all internal forces are obtained when the solutions (49) are substituted into (13), (14), and the last two equations of the elastic equation (8):

$$
\begin{aligned}
N_{1}= & Q_{1} \cot \alpha+N_{1}^{*}=\frac{\varepsilon E t^{2}}{R}\left(\Omega_{0}+\varepsilon \Omega_{1}+\varepsilon^{2} \Omega_{2}\right)+N_{1}^{*}, \\
N_{2}= & \frac{E t^{2}}{R \cos \alpha_{0}}\left(\frac{d \Omega_{0}}{d y}+\varepsilon \frac{d \Omega_{1}}{d y}+\varepsilon^{2} \frac{d \Omega_{2}}{d y}\right) \\
& -\frac{\varepsilon E t^{2} \cos \left(\alpha_{0}-\varphi\right)}{R \sin ^{2}\left(\alpha_{0}-\varphi\right)}\left(\Omega_{0}+\varepsilon \Omega_{1}+\varepsilon^{2} \Omega_{2}\right)+N_{2}^{*},
\end{aligned}
$$

$M_{1}$

$$
\begin{aligned}
=-\frac{D t}{R^{2}} & {\left[\frac{\sin ^{2}\left(\alpha_{0}-\varphi\right)}{\varepsilon^{2} \cos ^{2} \alpha_{0}}\left(\frac{d^{2} K_{0}}{d y^{2}}+\varepsilon \frac{d^{2} K_{1}}{d y^{2}}+\varepsilon^{2} \frac{d^{2} K_{2}}{d y^{2}}\right)\right.} \\
& \left.+(1+\mu) \frac{\cos \left(\alpha_{0}-\varphi\right)}{\varepsilon \cos \alpha_{0}}\left(\frac{d K_{0}}{d y}+\varepsilon \frac{d K_{1}}{d y}+\varepsilon^{2} \frac{d K_{2}}{d y}\right)\right],
\end{aligned}
$$

$M_{2}$

$$
\begin{aligned}
=-\frac{D t}{R^{2}} & {\left[\mu \frac{\sin ^{2}\left(\alpha_{0}-\varphi\right)}{\varepsilon^{2} \cos ^{2} \alpha_{0}}\left(\frac{d^{2} K_{0}}{d y^{2}}+\varepsilon \frac{d^{2} K_{1}}{d y^{2}}+\varepsilon^{2} \frac{d^{2} K_{2}}{d y^{2}}\right)\right.} \\
& \left.+(1+\mu) \frac{\cos \left(\alpha_{0}-\varphi\right)}{\varepsilon \cos \alpha_{0}}\left(\frac{d K_{0}}{d y}+\varepsilon \frac{d K_{1}}{d y}+\varepsilon^{2} \frac{d K_{2}}{d y}\right)\right] .
\end{aligned}
$$

\section{Case Studies}

In this section, the effect of material and geometric parameters on the nonlinear response of axisymmetric deep thin spherical shell under uniform external pressure is analyzed by an example. The shells are assumed to be clamped along boundary edge. In characterizing the behavior of the spherical shells, deformations in which the central region of a shell moves toward the plane that contains the periphery of the shell are referred to as inward deflections (positive deflections). Deformations in the opposite direction are referred to as outward deflections (negative deflections).

As part of the validation of the present method, the static behavior of a deep thin spherical shell under uniform external pressure is analyzed, which was also considered by Fan using

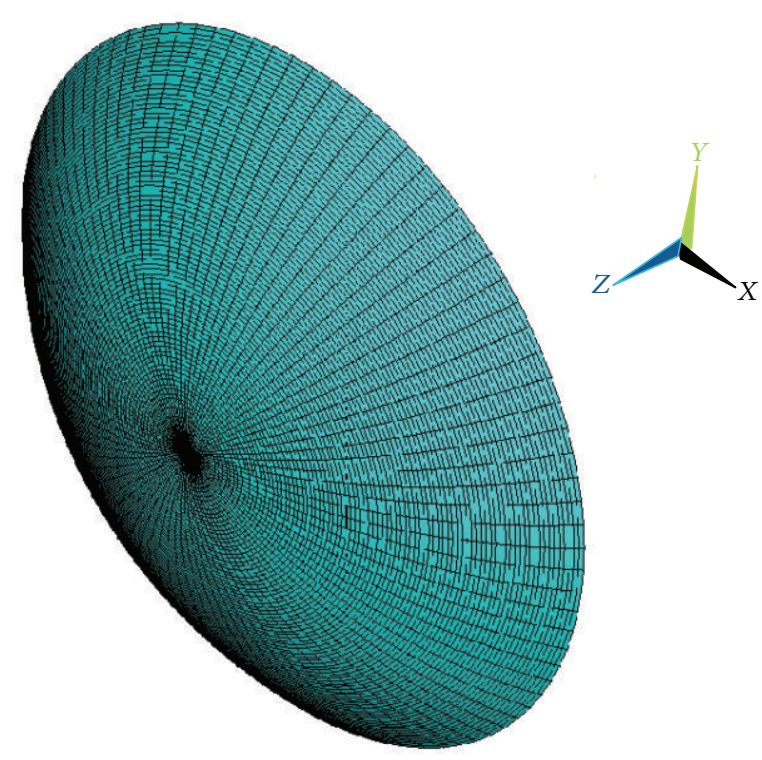

FIgURE 2: A finite element model of the deep thin spherical shell.

a differential equation with the assumption that the midsurface meridian displacement $u=0$ under the edge effect.

To illustrate the proposed approach, we consider a standard shell with the following properties: thickness $t=0.2 \mathrm{~m}$, spherical radius $R=10 \mathrm{~m}$, central angle $\alpha=45^{\circ}$, Young's modulus $E=30 \mathrm{GPa}$, and Poisson's ratio $\mu=0.3$.

To verify the accuracy of the perturbation solution, a finite element model of the deep thin spherical shell is established using ANSYS, which is shown in Figure 2. The effects of material and geometric parameters on the nonlinear response of the deep thin spherical shells with immovable clamped edge under uniform external pressure and the error analysis between ANSYS numerical solution and perturbation solution are considered in Figures 3 10. It should be noted that, in Figures 3(a) 10(a), the solid lines ((1), green color; (2), blue color; (3), red color) represent perturbation solution, and the dots ((1), green color; (2), blue color; (3), red color) represent ANSYS numerical solution.

Figures 3(a) and 4(a) show the effect of thickness $(t=$ $0.1,0.2$, and 0.3 ) on the nonlinear response of the deep thin spherical shells subjected to external pressure. As a whole, the absolute value of internal force $M_{1}$ increases when $t$ gets larger, whereas internal force $N_{1}$ changes in the opposite trend. Furthermore, through the comparison of the ANSYS numerical solution and perturbation solution, their changing tendency is in agreement qualitatively. The error analysis on the result obtained by using the two different ways in which the method of global error analysis is used to consider the effect of thickness on the deep thin spherical shells is conducted in Figures 3(b) and 4(b). The calculated results analysis shows that the error of the perturbation method can be controlled in a limited range, so we see the method does well in validity.

Figures 5(a) and 6(a) depict the effect of curvature radius ( $R=5,10$, and 15 ) on the nonlinear behavior of the externally 


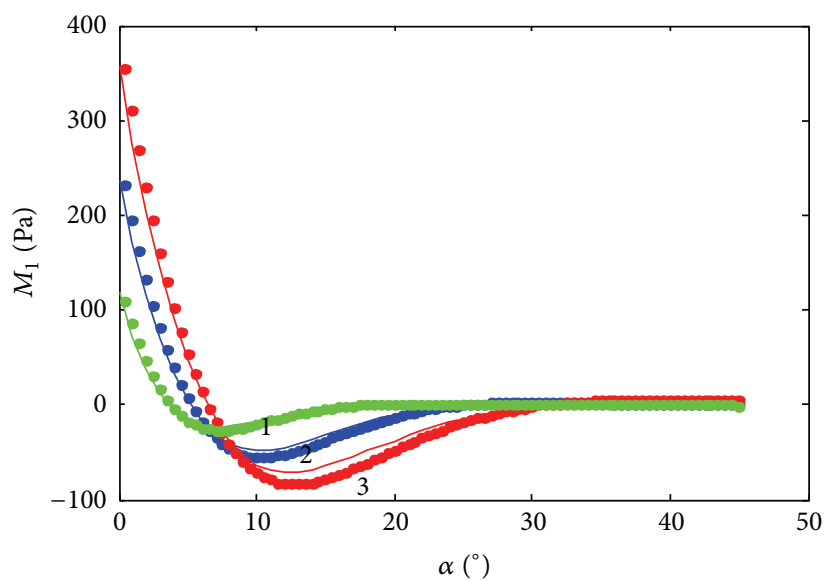

- Perturbation solution

- ANSYS numerical solution

(1) $t=0.1 \mathrm{~m}$

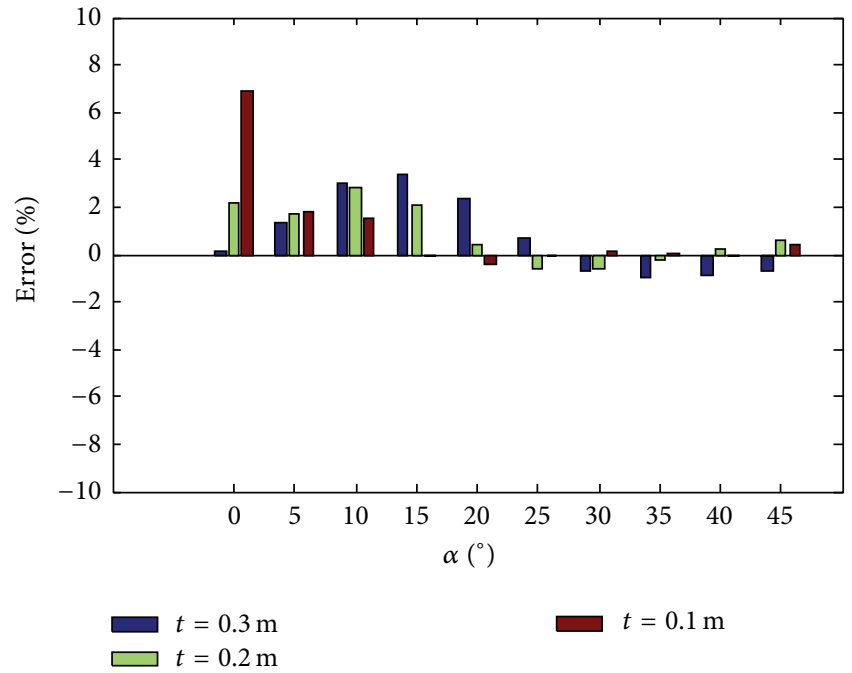

(b)

FIGURE 3: Effect of thickness $t$ on the internal force $M_{1}$ of the deep thin spherical shells.

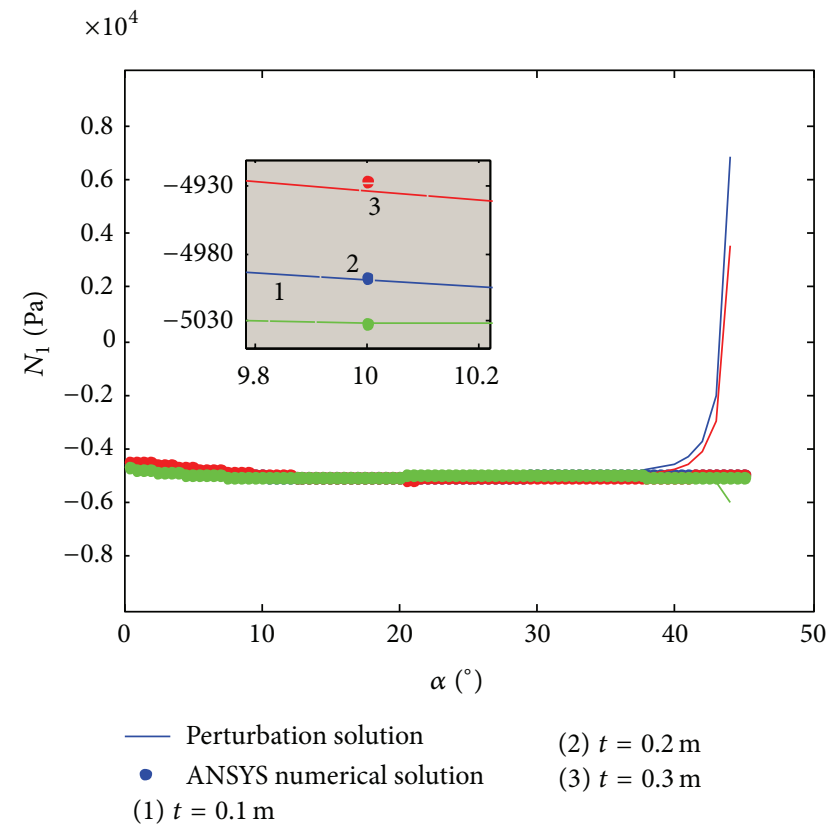

(a)

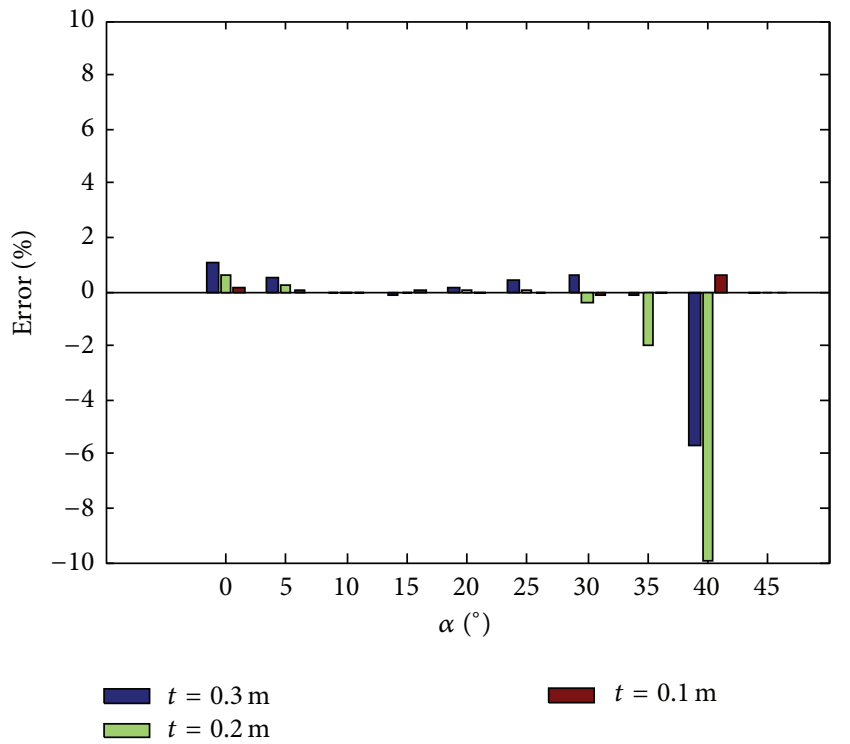

(b)

FIGURE 4: Effect of thickness $t$ on the internal force $N_{1}$ of the deep thin spherical shells.

pressurized deep thin spherical shells. As can be observed, the absolute value of internal forces $M_{1}$ and $N_{1}$ enhances considerably as curvature radius $R$ increases. In addition, a similar tendency exists in ANSYS numerical solution and perturbation solution. Figures 5(b) and 6(b) assess the global error between ANSYS numerical solution and perturbation solution. The largest difference is less than $5 \%$ in each figure, which means there is a good agreement with the two methods.
Figures 7 (a) and 8 (a) illustrate the effect of central angle $\left(\alpha=30^{\circ}, 45^{\circ}\right.$, and $60^{\circ}$ ) on the nonlinear response of deep thin spherical shells subjected to uniform external pressure. It is shown that the nonlinear response of spherical shells is not very sensitive with change of central angle $\alpha$ characterizing the deepness of spherical shell. Specifically, the enhancement of the internal forces $M_{1}$ and $N_{1}$ is small as the central angle $\alpha$ decreases. Basically, ANSYS numerical solution and perturbation solution change trends are consistent with 

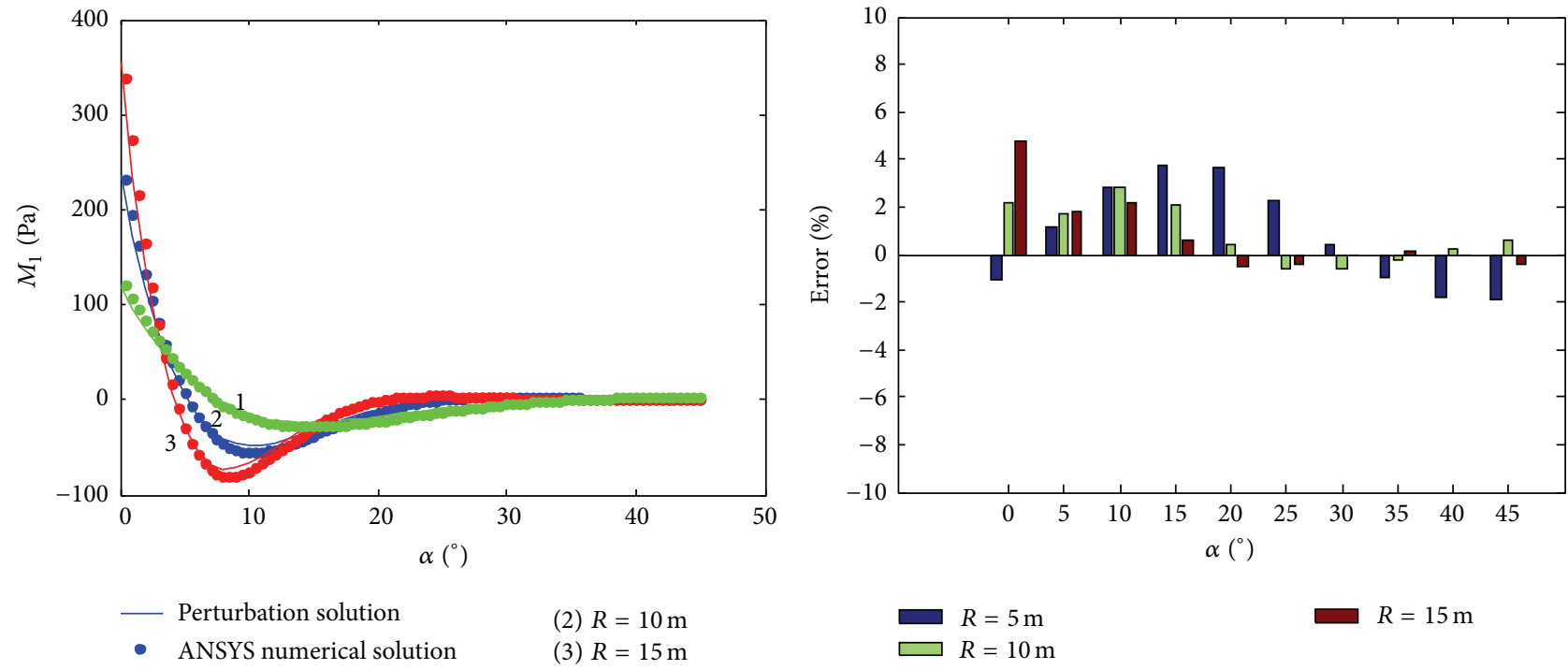

- ANSYS numerical solution

(1) $R=5 \mathrm{~m}$

(3) $R=15 \mathrm{~m}$

(b)

FIGURE 5: Effect of curvature radius $R$ on the internal force $M_{1}$ of the deep thin spherical shells.

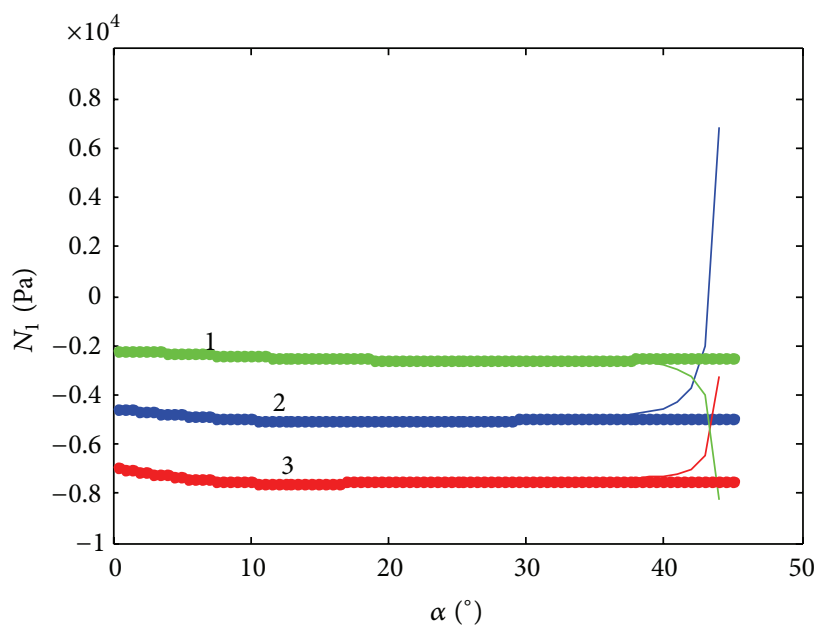

- Perturbation solution

- ANSYS numerical solution

(1) $R=5 \mathrm{~m}$

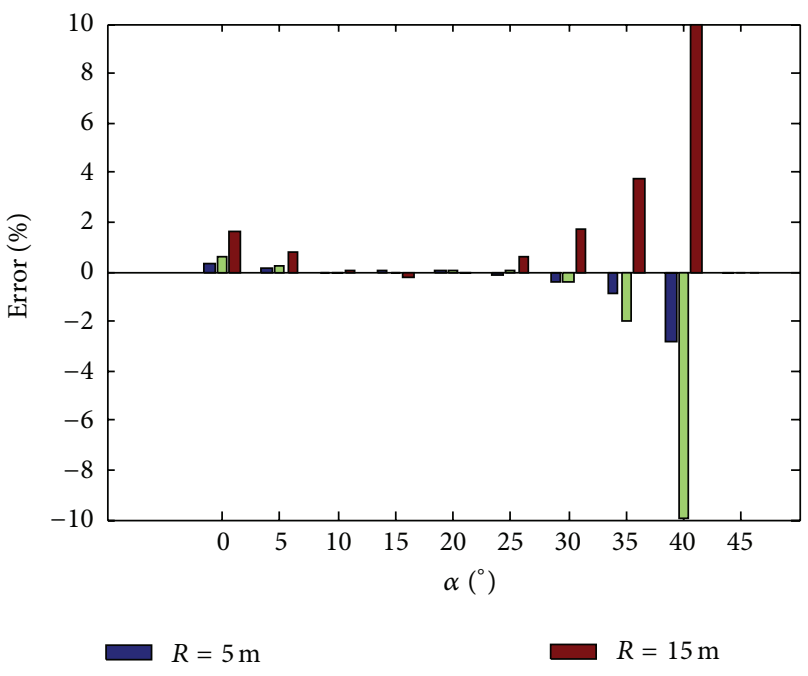

(b)

Figure 6: Effect of curvature radius $R$ on the internal force $N_{1}$ of the deep thin spherical shells.

the change of central angle. The global error between the numerical solution and the perturbation solution is analyzed in Figures 7(b) and 8(b). As can be inferred, the results of numerical solution and perturbation solution are very consistent since the maximum error is less than $10 \%$, so the creditability and rationality of the perturbation method are verified.

The effect of material parameters on the nonlinear response of deep thin spherical shells under external pressure is considered in Figures 9(a) and 10(a). In these figures the maximum internal force $M_{1}$ occurs in the shells made of concrete, followed by the steel shells, and the $M_{1}$ of the rubber shell is the smallest. On the contrary, the internal force $N_{1}$ shows an opposite tendency. In comparison, the change of perturbation solution is in close accord with that of numerical solution. A global error analysis is carried out on the result using the two different ways in Figures 9(b) and 10(b). As can be observed, the agreement with the numerical solution is fairly good. The global error between numerical solution and perturbation solution is not greater than $5 \%$.

Besides, in the figures which show the effect of geometric and material parameters on the internal forces $N_{1}$ of the deep 


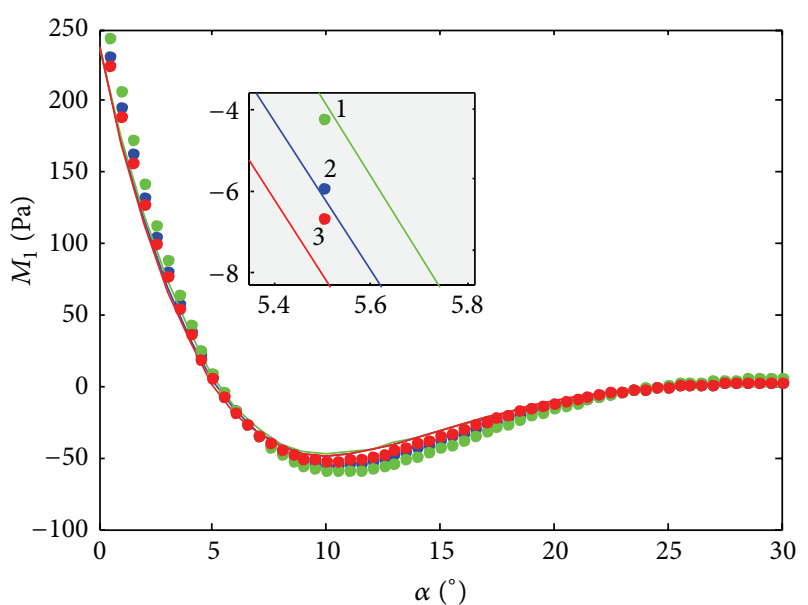

- Perturbation solution

- ANSYS numerical solution

(1) $\alpha=30^{\circ}$

(a)

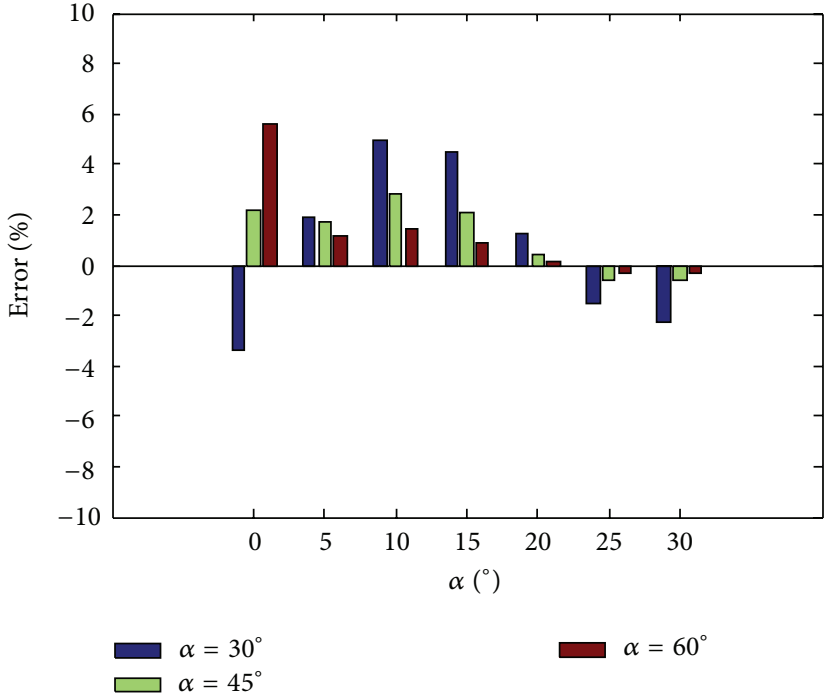

(b)

Figure 7: Effect of central angle $\alpha$ on the internal force $M_{1}$ of the deep thin spherical shells.

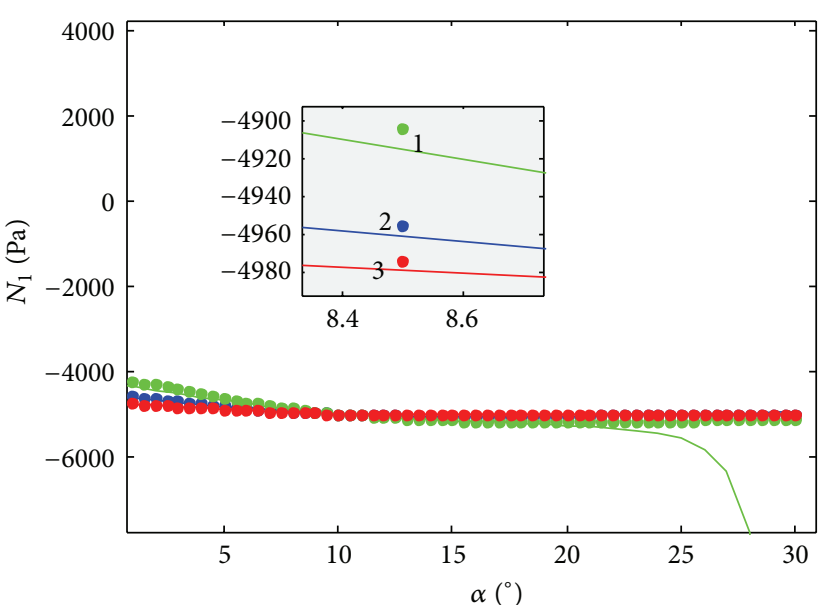

— Perturbation solution

- ANSYS numerical solution

(1) $\alpha=30^{\circ}$

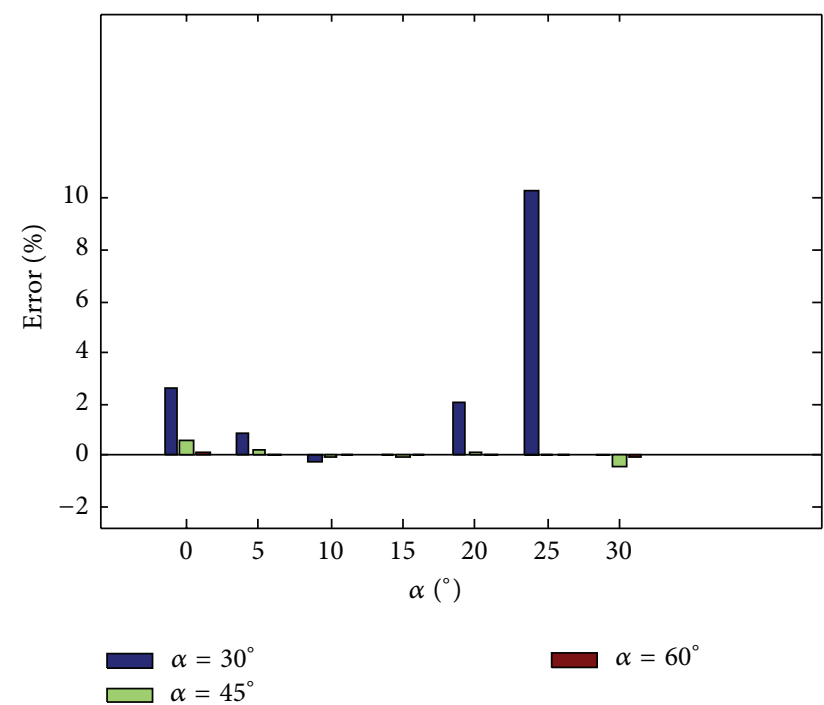

(b)

Figure 8: Effect of central angle $\alpha$ on the internal force $N_{1}$ of the deep thin spherical shells.

thin spherical shells, it is obvious that, near the top of the deep thin spherical shell, the internal force $N_{1}$ tends to infinity and the error is far beyond normal tolerance, which shows that the perturbation solution is not applicable near the top of the shells.

\section{Conclusions}

(1) On the basis of the Kirchhoff-Love hypothesis and the general theory of elastic thin shells, the author established a fundamental equation for a thin shell under the moment theory. Thus, it is not necessary to prove that the mid-surface meridian displacement $u=0$ under the edge effect. However, it is still feasible to employ a composite expansion to find the boundary value of axisymmetric bending deep thin spherical shells.

(2) The perturbation solution's internal forces are close to the numerical solutions by ANSYS, which means the method proposed in this paper does well in validity.

(3) The thickness $t$ and curvature radius $R$ have great effect on the internal forces $M_{1}$ and $N_{1}$ of the deep 


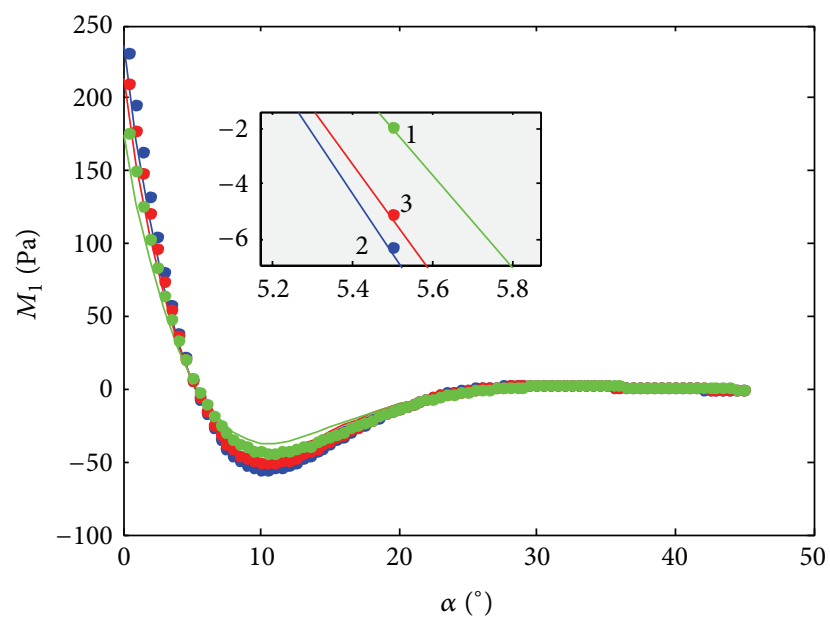

- Perturbation solution

- ANSYS numerical solution

(1) Rubber
(2) Concrete

(3) Steel

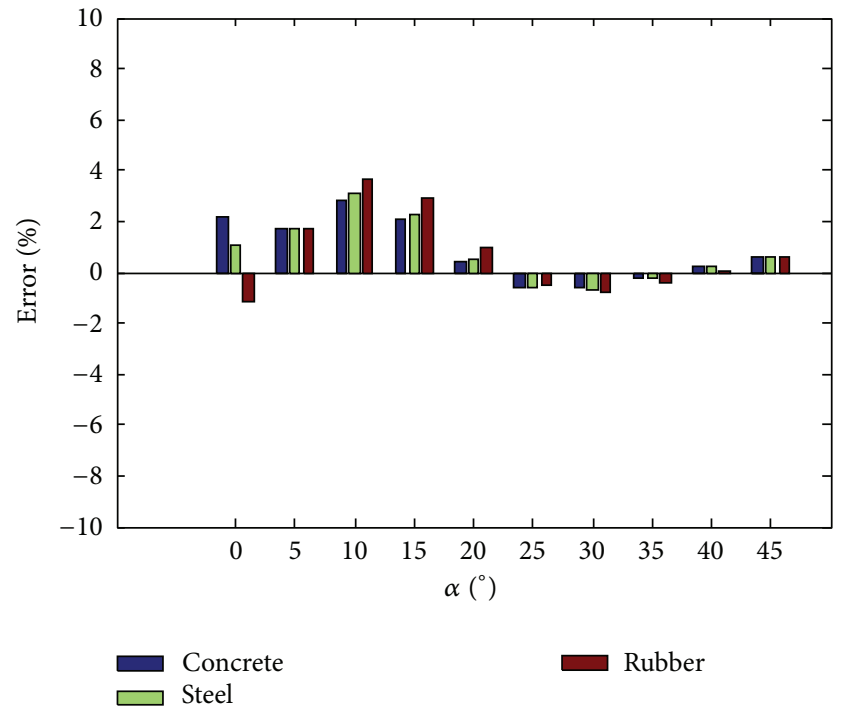

(b)

FiguRE 9: Effect of material parameters on the internal force $M_{1}$ of the deep thin spherical shells.

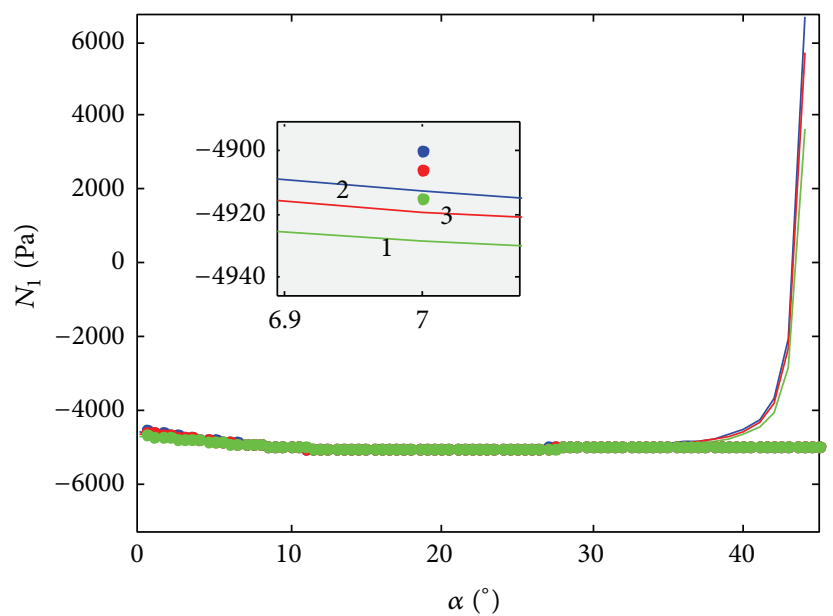

- Perturbation solution

- ANSYS numerical solution

(1) Rubber

(a)

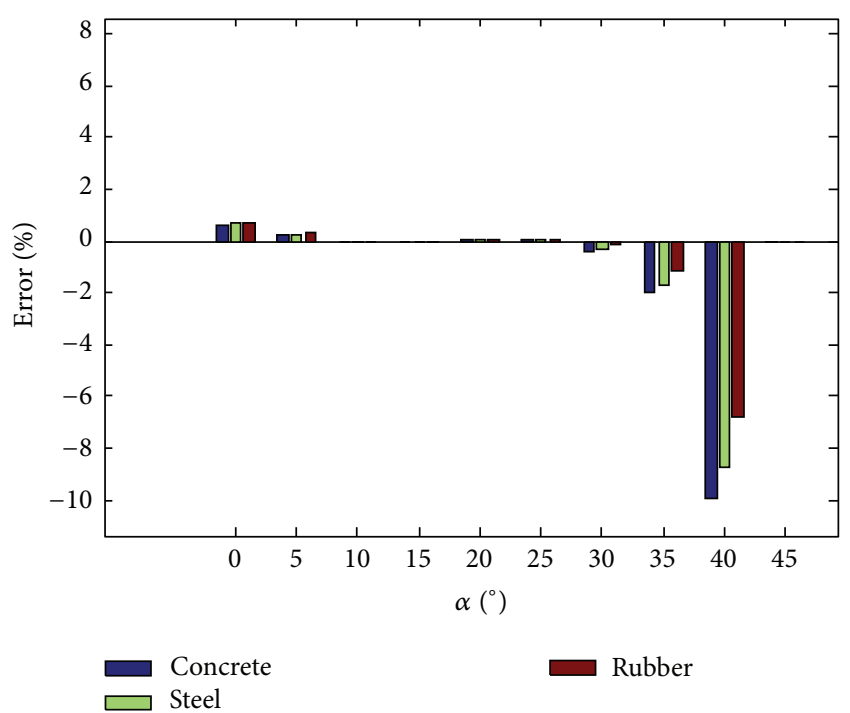

(b)

thin spherical shells, while the impact of the central angle $\alpha$ and material parameters on the internal forces $M_{1}$ and $N_{1}$ of the deep thin spherical shells is very small.

\section{Conflict of Interests}

The authors declare that there is no conflict of interests regarding the publication of this paper.

\section{Acknowledgments}

The authors gratefully acknowledge the financial support provided by the state high-tech research and development plans (863), Grant no. 2014AA110402, the Project of National Key Technology R\&D Program in the 12th Five-Year Plan of China (Grant no. 2012BAJ11B01), the National Nature Science Foundation of China (Grant no. 50978196), State Meteorological Administration Special Funds of Meteorological Industry Research (Grant no. 201306102), and the Fundamental Research Funds for the Central Universities. 


\section{References}

[1] D. Dan, R. Xiao, W. Bai, W. Cheng, and Y. Zhao, "A FBG based wind pressure sensor," China Patent ZL201320012780.2, 2013.

[2] Q. Weichang, "Asymptotic behavier of a thin clamped circular plate under uniform normal pressure at very large deflection," Science Report of Tsinghua University, vol. 1, pp. 1-24, 1948 (Chinese).

[3] S. Lukasiewicz, "Concentrated loads on shallow spherical shells," Quarterly Journal of Mechanics and Applied Mathematics, vol. 20, no. 3, pp. 293-305, 1967.

[4] D. Huy Bich and H. Van Tung, "Non-linear axisymmetric response of functionally graded shallow spherical shells under uniform external pressure including temperature effects," International Journal of Non-Linear Mechanics, vol. 46, no. 9, pp. 1195-1204, 2011.

[5] D. G. Ashwell, "On the large deflection of a spherical shell with an inward point load," in Proceedings of the Symposium on the Theory of Thin Elastic Shells, pp. 43-63, Delft, The Netherlands, 1959.

[6] G. V. Ranjan and C. R. Steele, "Large deflection of deep spherical shells under concentrated load," in Proceedings of the AIAAJ/ASME 18th Structures. Structural Dynamics and Materials Conference, Technical paper No. 77-411, pp. 269-278, San Diego, Calif, USA, 1977.

[7] Q. S. Li, J. Liu, and J. Tang, "Buckling of shallow spherical shells including the effects of transverse shear deformation," International Journal of Mechanical Sciences, vol. 45, no. 9, pp. 1519-1529, 2003.

[8] Z. Rusan, Theory of Elasticity, China Astronautic Publishing House, Beijing, China, 1992.

[9] E. Reissner, "On finite symmetrical deflections of thin shells of revolution," Journal Applied Mechanics, vol. 36, pp. 267-270, 1969.

[10] E. Reissner, "On finite symmetrical strain in thin shells of revolution," Journal of Applied Mechanics, vol. 39, no. 4, pp. 11371138, 1972.

[11] A. Y. Evkin and A. L. Kalamkarov, "Analysis of large deflection equilibrium states of composite shells of revolution. Part 1. General model and singular perturbation analysis," International Journal of Solids and Structures, vol. 38, no. 50-51, pp. 8961-8974, 2001.

[12] A. Y. Evkin and A. L. Kalamkarov, "Analysis of large deflection equilibrium states of composite shells of revolution. Part 2. Applications and numerical results," International Journal of Solids and Structures, vol. 38, no. 50-51, pp. 8975-8987, 2001.

[13] J. Thredgold, Mathematical modelling of the deformation of spectacle lenses [Ph.D. thesis], University of South Australia, 2007.

[14] L. Colgan, P. Howlett, and J. Thredgold, "A perturbation analysis to solve the linear equations for symmetric deformation of a deep spherical shell," Mathematical and Computer Modelling, vol. 49, no. 5-6, pp. 918-935, 2009.

[15] A. Y. Evkin, "Large deflections of deep orthotropic spherical shells under radial concentrated load: asymptotic solution," International Journal of Solids and Structures, vol. 42, no. 3-4, pp. 1173-1186, 2005.

[16] F. Cunxu, "The peripheral effect of the bend of axial symmetry for spherical shell," Engineering Mechanics, vol. 5, no. 4, pp. 1-6, 1988.

[17] C. X. Fan, "Refined differential equations of deflections in axial symmetrical bending problems of spherical shell and their singular perturbation solutions," Applied Mathematics and Mechanics, vol. 11, no. 12, pp. 1103-1112, 1990 (Chinese).

[18] C. Fan, "Conjugate second-order deflection differential equation of global shell axial symmetrical bending problem," Chinese Journal of Theoretical and Applied Mechanics, vol. 39, no. 5, pp. 704-707, 2007 (Chinese).

[19] N. El Meiche, A. Tounsi, N. Ziane, I. Mechab, and E. A. Adda.Bedia, "A new hyperbolic shear deformation theory for buckling and vibration of functionally graded sandwich plate," International Journal of Mechanical Sciences, vol. 53, no. 4, pp. 237-247, 2011.

[20] L. O. Larbi, A. Kaci, M. S. A. Houari, and A. Tounsi, "An efficient shear deformation beam theory based on neutral surface position for bending and free vibration of functionally graded beams," Mechanics Based Design of Structures and Machines, vol. 41, no. 4, pp. 421-433, 2013.

[21] A. Bessaim, M. S. A. Houari, A. Tounsi, S. R. Mahmoud, and E. A. Adda Bedia, "A new higher-order shear and normal deformation theory for the static and free vibration analysis of sandwich plates with functionally graded isotropic face sheets," Journal of Sandwich Structures and Materials, vol. 15, no. 6, pp. 671-703, 2013. 


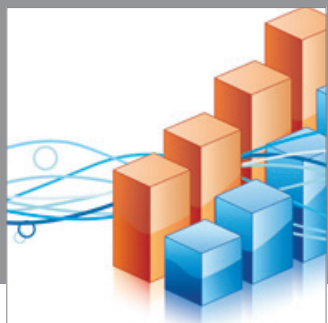

Advances in

Operations Research

mansans

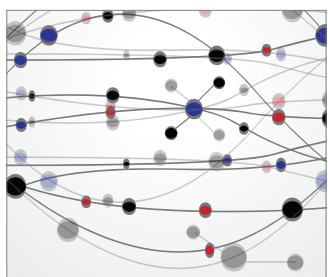

The Scientific World Journal
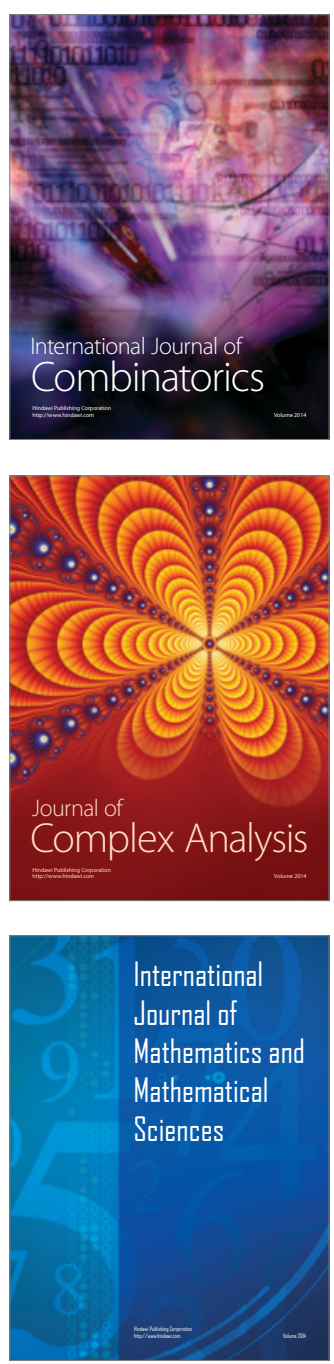
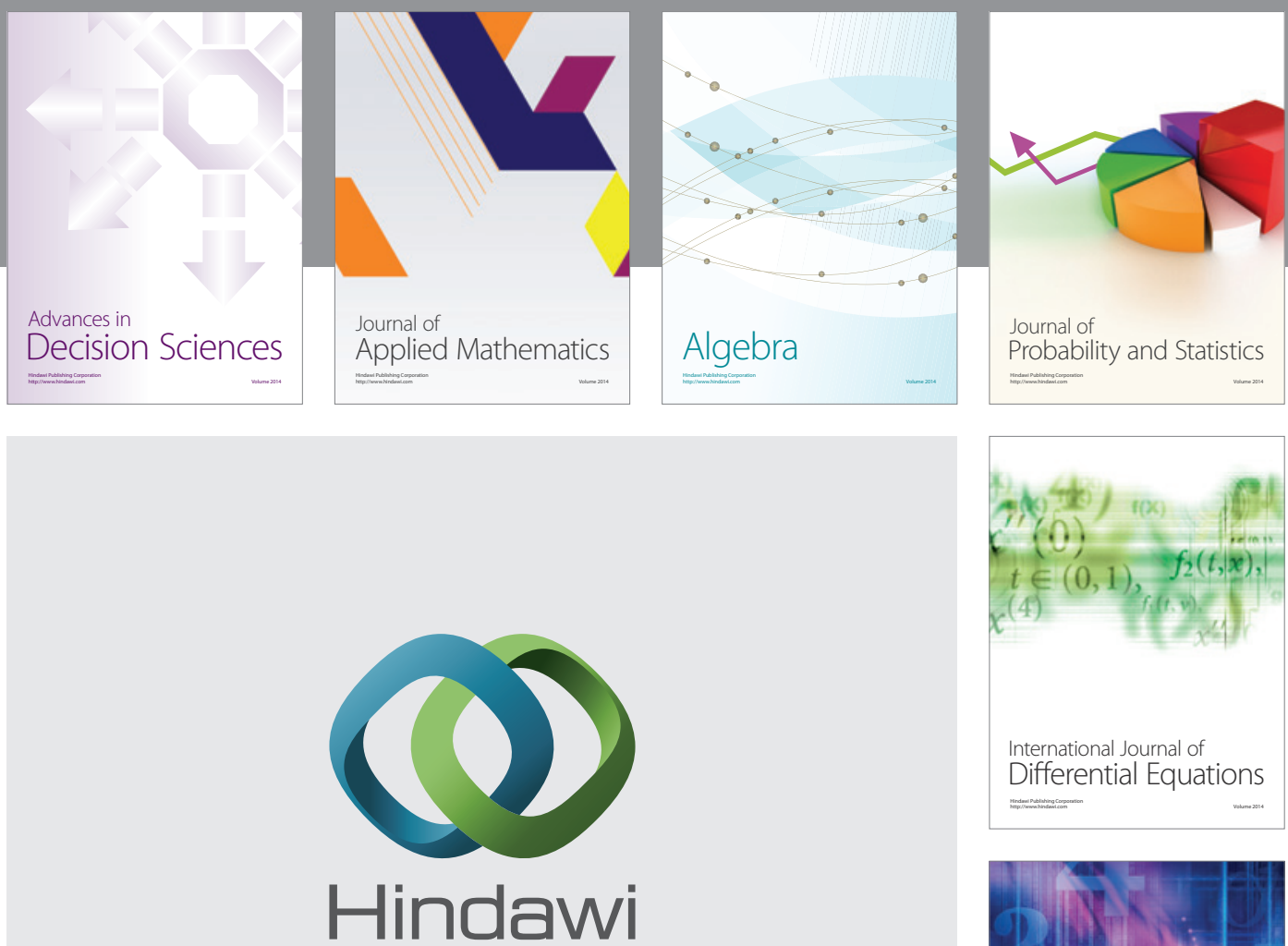

Submit your manuscripts at http://www.hindawi.com
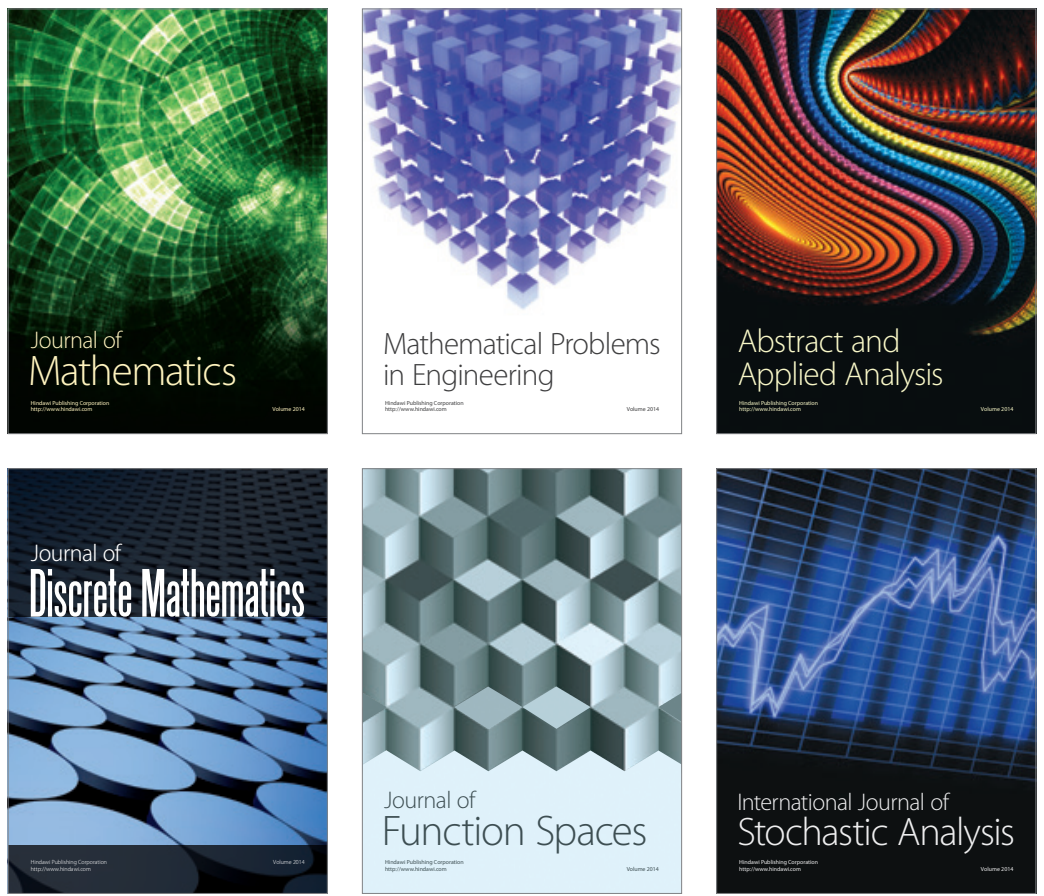

Journal of

Function Spaces

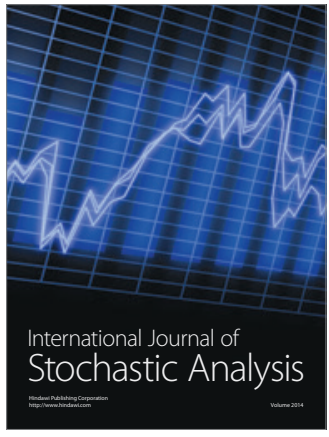

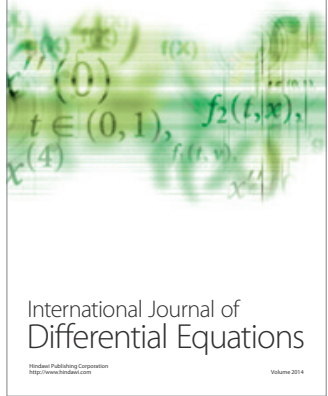
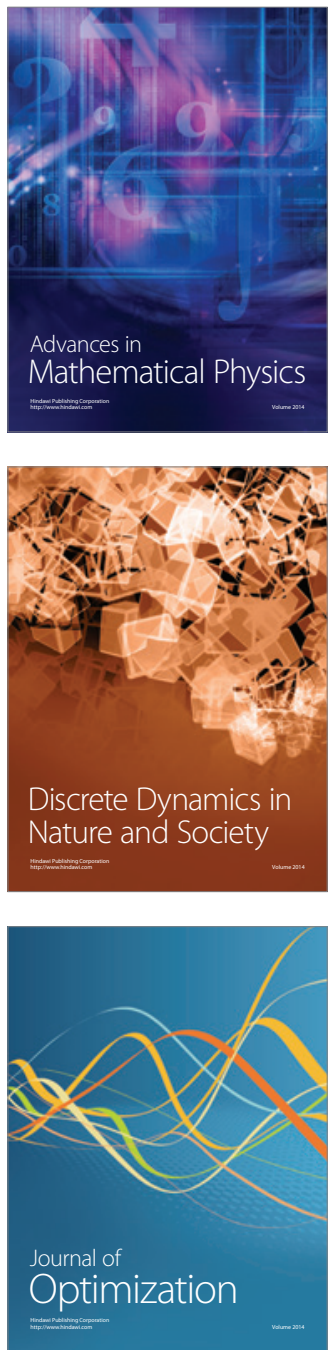